\title{
ANALYSIS OF MAGNETIC GROUND STATE IN THULIUM IRON GARNETS
}

\author{
A. Koper, A. Leimann-Szweykowska \\ Institute of Plyysics, A. Mickiewicz University, Matejki 48/49, 60-769 Poznań, Poland \\ P.E. Wigen and R.E. Bornfreund \\ Department of Physics, The Ohio State University, Columbus OH 43210 USA
}

Magnetic plase diagrams of thulium iron garnets at $T=0 \mathrm{~K}$ are obtained by means of the so-called Monte Carlo relaxation method. The diagrams are given in terms of the respective strength of the superexchange couplings. There are five different phases and one spin-flop line on the diagram obtained. Two of these phases are of a semi-spin glass type.

PACS numbers: 75.10.IIk, 75.40.Mg, 75.50.-y, 75.50.Gg, 75.50.Lk

\section{Introduction}

Thulium iron garnet (TmIG) and also its derivatives are known to display unusual magnetic bchaviour. A large number of questions still remain open. For instance, it has been recently reported that single-crystal TmIG thin films doped with bismuth and gallium undergo a continuous re-orientation of their easy axis of magnetization from the in-plane to the perpendicular to the plane [1, 2].

In TmIG the octahedral $[a]$ and tetrahedral $(d)$ sites are occupied by $\mathrm{Fe}^{3+}$ whose ground state is the orbital $S$ singlet, ${ }^{6} S_{5 / 2}$. Thus, the ferrous magnetism is purely spin-like in its nature. Thulium ions $\mathrm{Tm}^{3+}$ occupy the dodecahedral sublattice $c$. The ground state of $\mathrm{Tm}^{3+}$ is the multiplet, ${ }^{3} H_{6}$ with a large orbital contribution to the total angular momentum operator, $J$. The overall action of the crystal field which at the $c$ sites is considered to be cubic with a trigonal distortion, on the thulium magnetic moments produces a considerable reduction of their large values $(J=6)$. The total angular momentum of $\mathrm{Tm}^{3+}$ projected on the lowest-lying crystal-field energy level, is reduced to that of one [3].

In order to understand the magnetic properties of both pure and doped TmIG, it is necessary to determine a magnetic ground state of the system in a wide range of its superexchange parameters. A three-sublattice Heisenberg Hamiltonian is assumed with the superexchange nearest-neighbour interactions: $a-a, a-d, d-d$ and $d-c$. The $a-c$ and $c-c$ are considered negligibly small. 


\section{Determination of the magnetic ground state}

The magnetic ground state of the model is determined in the quasi-classical appr :-imation, where both the spin operators at the iron sites $(S=5 / 2)$ and the pseudo-spin operators at the sites $c(s=1)$ are substituted by classical vectors. The so-called relaxation Monte Carlo method is applied to 64 classical spins of the unit cell with the periodic boundary conditions imposed on the superexchange interactions $[4,5]$. In the case of quantum systems, the method is equivalent to the version of MFA, where for each lattice site the molecular field equation i. solved separately. The magnetic diagrams are obtained in the phase space of the suserex-

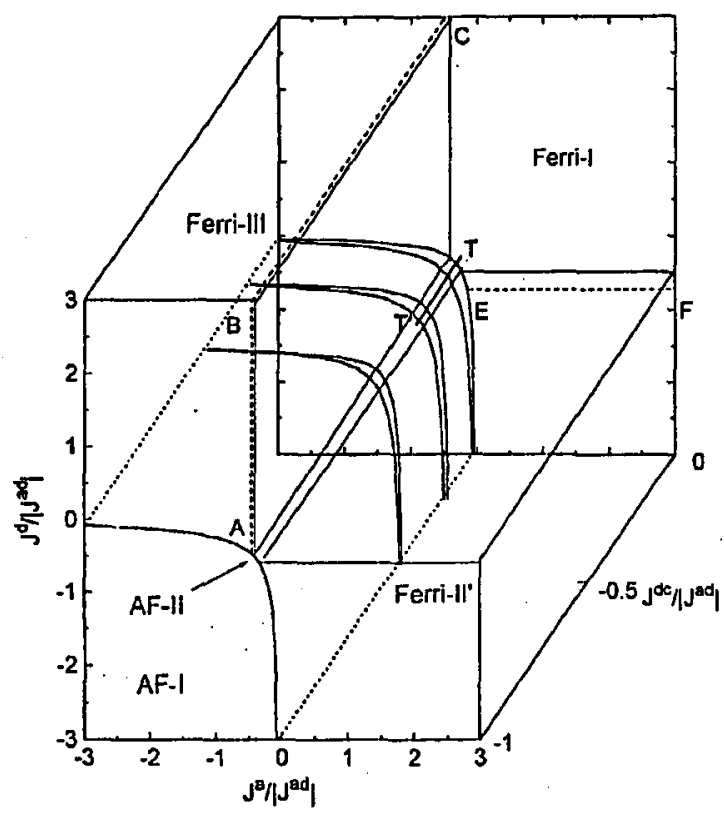

Fig. 1. The three-dimensional phase diagram of TmIG. Although the exact values of the superexchange integrals in TmIG are not known, they lie on the $T-T^{\prime}$ line.

change coupling parameters $J_{a}, J_{d}, J_{a d}$ and $J_{d c}$. Actually, the phase diagrams are three-dimensional (see Fig. 1). The dimension of the space is reduced by choosing the ratios: $J_{a} /\left|J_{a d}\right|, J_{d} /\left|J_{a d}\right|$ and $J_{d c} /\left|J_{a d}\right|$ as parameters. To get a better insight into the result, we perform a cut for certain values of $J_{d c}$ as shown in Fig. 2. For $J_{d c} /\left|J_{a d}\right|=-0.1$, the phase plane is divided into the following regions:

(1) Ferri-I is the only fully collinear region with a ferromagnetic ordering within each of the three sublattices. The magnetizations of the octahedral iron and thulium sublattices are parallel, whereas the tetraledral spins are in the antiparallel direction. A non-zero resultant magnetization is apparent. This phase is experimentally observed in TmIG. The diagram shows the position corresponding 


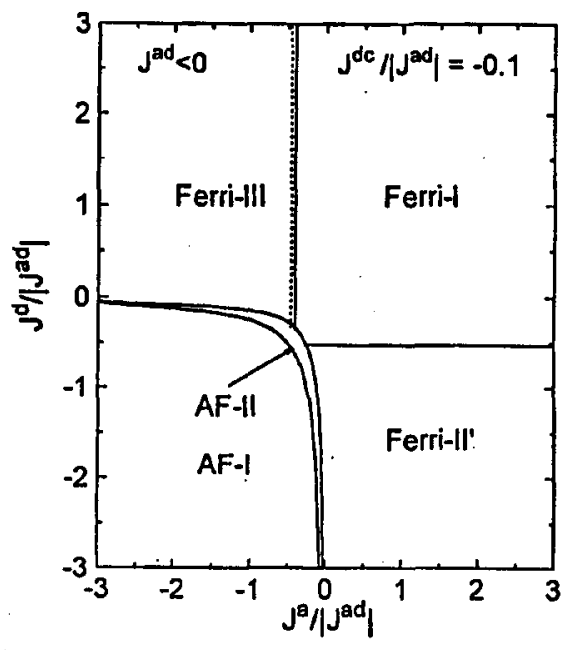

Fig. 2. The cut $\left(J_{d c} /\left|J_{a d}\right|=-0.1\right)$ of the phase diagram of TmIG.

to the values of the exchange parameters which were found to fit the experimental data (point $T$ ) [3].

(2) Ferri-II': magnetic moments of the $a$ and $c$ sublattices remain parallel, whereas the tctraliedral spins are in one plane but oriented at different angles to one another. The resultant magnetization is non-zero.

(3) Ferri-III: the roles of $a$ and $d$ sublattices are reversed with respect to those in the ferri-II' phase. There is also a spin-flop line in this region.

(1) $A F-I$ and AF-II: $a, d$ and $c$ sublattices have their resultant magnetizations equal to zero. In $\Lambda F-I$, the three mutually independent antiferromagnetic orderings lie in the same plane. The non-trivial infinite degeneracy of this phase results from indefinite position of the $a, d$ and $c$ orderings with respect to one another. In AF-II the situation is even more complex as the three antiferromagnetic orderings are arbitrarily oriented in the three-dimensional space.

It seems that a magnetic ground state of the system can be changed relatively easily since the "experimental" point $T$ as indicated both in Fig. 1 and 2 lies very close to the boundaries of the four phases, and especially to the spin-flop line. Since the magnetic properties are sensitive to doping, certain experimentally observed anomalies can hopefully be explained on the basis of this diagram. The influence of the third (thulium) sublattice is best seen in the three-dimensional diagram of Fig. 1, where for example, the "thickness" of the AF-II region changes with varying $J_{d c}$.

This work is supported by the Committee for Scientific Research (Poland) under grant No. 3 P408 00104. 


\section{References}

[1] R.E. Bornfreund, Ph.D. Thesis, Ohio State University, Ohio 1996.

[2] R.E. Bornfreund, D.C. Khan, P.E. Wigen, M. Pardavi-IIorvath, J.B. Ings, R.F. Belt, IEEE Trans. Magn. 28, 2991 (1992).

[3] A. Lehmann-Szweykowska, P.E. Wigen, L. Kowalewski, M.M. Kaczmarek, T.B. Mitchell, Phys. Rev. B 37, 459 (1988).

[4] L.R. Walker, R.E. Walstedt, Phys. Rev. Lett. 38, 514 (1977).

[5] A. Koper, A. Lehmann-Szweykowska, R. Wojciechowski, M. Mucha, J. Phys., Condens. Matter 7, 1391 (1995). 